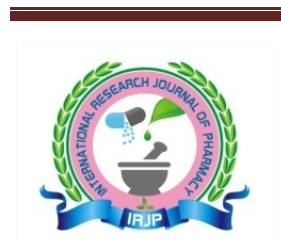

INTERNATIONAL RESEARCH JOURNAL OF PHARMACY

www.irjponline.com

ISSN $2230-8407$

\title{
Research Article \\ DEVELOPMENT AND IN VITRO CHARACTERIZATION OF NANOEMULSION AS ADJUVANT FOR
THE ADMINISTRATION OF VACCINE USING A MODEL ANTIGEN
}

R. Vijaya *, G. Aswini and S. Madheshwaran

Department of Pharmaceutical Technology, Anna University, BIT Campus, Tiruchirappalli- 620 024, Tamilnadu, India

*Corresponding Author Email: vrssvrs@gmail.com

Article Received on: 13/05/16 Revised on: 03/07/16 Approved for publication: 12/07/16

DOI: $10.7897 / 2230-8407.07783$

\begin{abstract}
The present study has investigated the development of oil in water type nanoemulsion (NE) for the incorporation of vaccine. NE act as adjuvant and potentiate the immunogenicity of antigen. The NE was formulated using plant essential oil, surfactant tween 80 and co surfactant ethanol by high energy emulsification method. The formulations were optimized for surfactant and cosurfactant ratio, $\mathrm{S}_{\text {mix }}$ concentration and a ternary phase diagram was constructed to identify the area of NE formation. Formulations of NE1 to NE5 were prepared by sonication which are subjected to varying number of sonication cycles to get NE in $100 \mathrm{~nm}-200 \mathrm{~nm}$ range and NE was subjected to short term and long term stability assessment. The NE was characterized in vitro for transparency, droplet size, zeta potential, $\mathrm{pH}$, viscosity, conductivity, surface tension, fourier transform infrared spectroscopy. Transparent stable nanoemulsion (NE5) having mean droplet diameter of $84.23 \mathrm{~nm}$ was obtained at $3: 2$ ratio of $\mathrm{S}_{\text {mix, }}(10 \%)$ and the oil and surfactant ratio of 1:5 (v/v). No significant change in droplet diameter was observed after one week of storage at different temperatures. Surfactant concentration has been found to play a vital role in obtaining a stable nanosized emulsion. Further the NE5 was incorporated with pertussis toxin (PTAg-NE5) as a model vaccine and analysed for compatibility, size and stability. The PTAg-NE5 showed the incorporation of antigen with a slight increase in size of $99.42 \mathrm{~nm}$. The developed NE was found to be suitable for the incorporation of vaccine and thus could potentiate the immunogenicity of antigen.
\end{abstract}

Keywords: Nanoemulsion, Formulation, In vitro characterization, Stability, Vaccine adjuvant, Antigen.

\section{INTRODUCTION}

Vaccines are long been effectively and economically used against many infectious diseases. Most of which are administered topically via injection into intramuscular, subcutaneous \& intradermal site in early childhood stage which produces pain and other side effects that leave the people with mental agony and stress. Needle less jet injections are available as an alternate to this problem. However the cost of such injection is very high \& is not affordable for economic countries. Hence formulation development approaches have been made to improve the safety, efficacy and convenient means of its administration. In the formulation of vaccine, adjuvant plays a significant role in inducing the immunogenicity of antigen. Among the several adjuvants employed, emulsion type of adjuvant like freund's complete adjuvant are most widely used because of their effective interaction with the antigen and their potential to form granulomas at the injection site which are well recognized by the immune cells present in our human body.

With the advent of nanotechnology, vaccine formulations has gained lot of benefits. Nano particulates offers in large surface area, surface free energy, sustained \& targeted delivery of therapeutics that provides protective \& effective immunity. Further these nanocarriers can be charged and have hydrophobic (or) receptor interacting properties that leads to better interaction with antigen presenting cells. Further nano vaccines are noninvasive, allowing painless delivery. Scientists started developing numerous therapeutic vaccines using nanotechnology against all kinds of antigens. One such novel formulation have been shown significant vaccine adjuvant activity is nanoemulsion (NE). In addition to its vaccine adjuvant effect it acts as a versatile delivery carrier, skin penetration enhancer, biocompatible formulation, protective for the antigen from degradation, targeted carrier, reduced dose of drug, thermodynamically stable, etc. The NE act as an effective adjuvant for vaccines by binding with the cell membranes of microbes and potentiate their action without affecting normal healthy cells. They have been reported to produce a strong and long lasting immunity through the induction of mucosal, systemic and cellular immunity. They help uptake by antigen presenting cells present in the skin, mucosa ${ }^{1}$ etc. As they have low surfactant concentration, they are non skin irritating with lots of energy. Further their long term stability does not necessitate the use of cold chain storage for vaccine formulation ${ }^{2}$. These NE's have been successfully adopted for mucosal and transdermal immunization ${ }^{3}$. However the immunogenicity greatly rely on the development of $\mathrm{NE}$ formulation. Many of the developed NE's have focused on mucosal immunity rather than the topical/transdermal immunization ${ }^{4}$. Human skin contains abundant dendritic and Langerhans cells which acts as antigen presenting cells in the immunization process. NE are found to penetrate effectively across the skin and through the hair follicles. Hence an attempt has been made for the development of o/w type NE suitable for the topical/transdermal delivery of antigen. Here, pertussis toxin (Bordetella pertussis) has been used as a model antigen. Pertusis vaccine is a subunit vaccine \& administered intramuscularly along with adjuvant as part of DTap combined immunization to prevent the occurrence of whooping cough in children. Generally, the intramuscular administration of adjuvanted vaccines are done to reduce the local adverse effects due to hyper activation of immune system. Here the development of $\mathrm{NE}$ as adjuvant for topical administration may reduce the risk of 
local adverse effects by their diffusion across the skin layers allowing slow release of antigen which could be processed effectively by the APC'S such as Langerhans cells \& dendritic cells present in skin layers.

\section{MATERIALS AND METHODS}

Soybean oil was obtained from Fortune refined oil, India. Tween 80 and ethanol were purchased from SDFCL, Mumbai- Sodium Chloride and Potassium Chloride were bought from Loba chemie, Mumbai. Disodium hydrogen phosphate, Potassium dihydrogen phosphate and Sodium Hydroxide were obtained from Merck specialties pvt ltd, Mumbai.

\section{Compatibility determination}

Compatibility between the formulation ingredients was tested by FTIR (Fourier Transform Infrared Spectroscopy (Jasco 6300 series, Jasco). For this the FTTR spectra for soya bean oil, Tween80, formulation NE5 \& 5\% PTAg-NE5 were taken. Spectra were recorded from 4000 to $650 \mathrm{~cm}^{-1}$ scanning range and the observed spectrum were compared to determine the compatibility.

\section{Formulation optimization of nanoemulsion}

NE formulation optimization was focused with respect to the selection of oil, surfactant, co-surfactant and their ratio/concentration. Among the various oils such as soybean oil, castor oil, clove oil and eucalyptus oil found from the literature regarding the formulation of $\mathrm{NE}$, soya bean oil having a viscosity of $1.572 \mathrm{mPas}$ was chosen as the oil phase ${ }^{5}$. It has been said that the droplet size of emulsion gets decreased when emulsion viscosity is increased ${ }^{6}$. Based on the HLB (Hydrophilic Lipophilic Balance) value tween 80 and ethanol were used as surfactant and co-surfactant. Surfactant and cosurfactant ratio was optimized for various ratios i.e. 1:1, 1:2, 1:3, $1: 4,4: 1,3: 1,2: 1,3: 2,3: 1$. The ratio of $3: 2$ and $10 \% \mathrm{v} / \mathrm{v}$ concentration of Smix (mixture of surfactant \& co-surfactant ) was concluded to be ideal for the formulation of transparent stable soybean oil NE. Less than $10 \% \mathrm{v} / \mathrm{v}$ concentration of Smix (i.e. $5 \%$ and $8 \%$ ) has resulted in the formation of unstable NE.

A pseudo ternary phase diagram was constructed using 3:2 ratio of $\mathrm{S}_{\text {mix }}$ by mixing different proportions of soya bean oil and aqueous phase. The mixture was gently stirred in a vortex mixer (Himedia, India) and kept at ambient temperature $\left(25^{\circ} \mathrm{C}\right)$ to attain equilibrium. The equilibrated samples were visually examined and determined as being clear and transparent nanoemulsion, or crude emulsions or gels. The physical state of clarity was represented on a pseudo-ternary phase diagram with one axis representing water, one representing oil and the third representing the Smix. The influence of weight ratio of surfactant to co-surfactant on the area of $\mathrm{o} / \mathrm{w}$ nanoemulsion region was investigated on the pseudo-ternary phase diagram using online free version of (Figure 1) prism ternary diagram software.

\section{Preparation of Nanoemulsion}

The NE was prepared by high energy emulsification technique ${ }^{7}$, ${ }^{8,9}$ using a bath sonicator (Citizen pvt. Ltd., Chennai). The composition of NE is given in table.1.

Primary emulsion was formed by agitating soybean oil and $\mathrm{S}_{\mathrm{mix}}$ (3:2) ratio thoroughly in a magnetic stirrer (Remi equipment Pvt Ltd, Mumbai) followed by water in drop wise manner with a help of a insulin syringe. The emulsion was maintained under magnetic stirring at $2000 \mathrm{rpm}$ for $45 \mathrm{~min}$ in order to get a stable preparation. It was then placed in a bath sonicator (Citizen Pvt ltd, Chennai) to size reduce the globules. Sonication was done for $8 \mathrm{~min}$ which is considered as 1pass (or cycle). Based on number of sonication cycle different formulations such as NE1 to NE5 were prepared. NE1=1pass, NE2-2 passes, NE3-3passes, NE4-4passes, NE5-4 passes

\section{Addition of antigen in nanoemulsion}

$2 \mathrm{ml}(84 \mu \mathrm{g} / \mathrm{ml})$ of pertusis toxin as a model antigen was incorporated into the prepared stable nanoemulsion $(5 \%$ - PTAgNE5) in the presence of phosphate buffer saline to maintain the $\mathrm{pH}$ in order to find its suitability. The composition of this formulation PTAg-NE5 is given in table 2.

\section{CHARACTERIZATION}

Transparency,
determination $^{10}$

The transparency of the NE formulation was tested visually for clarity against a dark background. The apparent $\mathrm{pH}$ was measured using a calibrated $\mathrm{pH}$ meter (Sartorius, Germany) at $25^{\circ} \mathrm{C}$. The $\mathrm{pH}$ was determined by dipping a glass electrode in to the formulation. Capillary viscometer (Sartorius, Germany) was used to assess the NE viscosity. For this $10 \mathrm{ml}$ of nanoemulsion was poured into the filling tube and transferred into the capillary tube by gentle suction. The time was recorded in seconds for the flow of liquid from the upper to lower mark in the capillary tube. It was generally expressed in cp. Electrical conductivity was measured using a conductivity meter (Borosil ltd, Mumbai) having a cell constant of $0.11 \mathrm{~cm}^{-1}$ at the frequency of $94 \mathrm{~Hz}$ and the measurements were carried out at $25 \pm 1^{\circ} \mathrm{C}$. The surface tension was determined using a stalagmometer (Borosil ltd, Mumbai) by drop count method. In this method, the formulation was sucked into the drop pipette up to a mark. Keeping the pipette vertically, the number of drops formed when the liquid level falls was noted. The relative surface tension of the samples were calculated using the formula Surface tension = $\mathrm{dl} * \mathrm{nw} / \mathrm{dw}^{*} \mathrm{nl}, \mathrm{dl}=$ Density of formulation, $\mathrm{nw}=$ Viscosity of water, $\mathrm{dw}=$ Density of water, $\mathrm{nl}=$ Viscosity of formulation.

\section{Droplet size and zeta potential}

Size and zeta potential of NE was measured using a Malvern zeta sizer (Malvern Ver. 20, Germany). $1 \mathrm{ml}$ of the formulation was taken in a glass cuvette and placed in the instrument for particle size analysis and zeta potential determination.

The electrophoretic mobility was obtained by Malvern zetsizer instrument after diluting suitable quantity of sample with $5 \mathrm{ml}$ of filtered water followed by injection in to electrophoretic cell. The zeta potential value was calculated using the instrument software with the help of smoluchosky equation. The magnitude of the zeta potential values gives an indication about the stability of NE formulation.

\section{Transmission Electron Microscopy analysis}

The morphology of the prepared vaccine of (PTAg-NE5) was studied using Transmission Electron Microscopy (TEM) (TECNAI 10 (Philips), Japan) operated at 70-80kv at $54700 \mathrm{x}$ magnification. To perform the TEM observations, the formulation was very well diluted with distilled water. A drop of the diluted sample was directly deposited on a carbon-coated grid, allowed to dry and observed. In addition, droplet sizes 
were measured using TEM by point-to-point resolution technique.

\section{STABILITY STUDIES}

Stability studies are highly required for emulsion kind of formulation, as because the changes in environmental stress conditions particularly temperature may influence the solubility of NE components that renders the formulation become unstable or less stable. The stability findings are helpful in determining the shelf storage of formulation. In order to hasten the process of stability testing, few of the methods have been reported in the literature. The methods of centrifugation, accelerated stability testing have been adopted to assess the long term stability of pharmaceutical formulations ${ }^{7}$.

\section{Centrifugation}

This method has been adopted by scientist to assess the short term stability of formulations in a laboratory scale.The optimized NE of NE5 was subjected to short term stability testing by centrifugation (REMI Sales \& Engineering Ltd, New Delhi) at $10000 \mathrm{rpm}$ for $30 \mathrm{~min}$ at $27^{\circ} \mathrm{C}$. The stability was assessed in terms of phase separation in NE after centrifugation.

\section{Accelerated stability testing}

The long term stability of pharmaceutical formulations are usually tested by accelerated testing which involves acceleration of various stress conditions such as temperature \& humidity according to ICH guidelines. Here, the samples were kept at accelerated temperature conditions of $25 \pm 2,40 \pm 2$ and $5 \pm 2$ maintained in a stability testing chamber (Wadegati lab equipment's Pvt. Ltd., India). The samples were analyzed for droplet size, $\mathrm{pH}$ and electrical conductivity after a storage period of $24 \mathrm{~h}, 3$ days and 7 days.

\section{STATISTICAL ANALYSIS}

The experimental values represent the average of six determinations. The calculated standard deviation values were subjected to ANOVA to find the statistical difference among the formulations. The significance value of $\mathrm{P}<0.05$ was kept for all the determinations.

\section{RESULTS AND DISCUSSION}

The FTIR spectra for soya bean oil, tween 80, NE5 and PTAgNE5 are given in Figure 2. In the case of soyabean oil, a triglyceride that are an ester derivative ensures two strong absorption peaks at $1745 \mathrm{~cm}^{-1}$ and $1163 \mathrm{~cm}^{-1}$ due to $\mathrm{C}=\mathrm{O}$ and $\mathrm{C}$ $\mathrm{O}-\mathrm{C}$ stretching and $2925 \mathrm{~cm}^{-1}$ due to $\mathrm{CH}$ stretching vibration. Tween 80 absorption bands appears at $2922 \mathrm{~cm}^{-1}, 1105 \mathrm{~cm}^{-1}$ and $1735 \mathrm{~cm}^{-1}$ are related to $-\mathrm{CH}_{2}, \mathrm{C}-\mathrm{O}-\mathrm{C}$ and $\mathrm{C}=\mathrm{O}$ respectively. The PTAg-NE5 spectra shows peaks at $3440 \mathrm{~cm}^{-1}$ owing to $\mathrm{OH}$ stretching vibration and peak at $1636 \mathrm{~cm}^{-1} 1$ confirms the $\mathrm{C}=\mathrm{O}$ stretching vibration. In case of FTIR spectra of NE5 \& PTAgNE5, no additional peaks were observed which indicates the compatibility of antigen with the ingredients of NE5 formulation. The FTIR of NE5 clearly displayed the interaction between tween 80 \& soybean oil that have resulted in the formation of NE. The absorption peaks for $\mathrm{C}=\mathrm{O}, \mathrm{C}-\mathrm{O}-\mathrm{C}$ stretching vibrations present in NE5 shows the internalization of soyabean oil by tween $80 \&$ the merging of polar and non polar regions with each other.

Among the various nanoemulsions (NE1 to NE5) prepared, formulation NE5 was appeared clear and transparent \& the photograph is shown in Figure 3. The pertussis mixed NE i.e PTAg-NE5 formulation is given in Table 2. Properties such as zeta size, $\mathrm{pH}$, viscosity, conductivity \& surface tension determined for the prepared nanoemulsions are presented in Table 3.

In the formulation of NE, co-surfactant plays a significant role in reducing the size of internal oil globules (o/w emulsion). This size reduction might be due to the rapid diffusion of cosurfactant ${ }^{11}$. Another criteria is the right choice of surfactant $\&$ co-surfactant which determines the HLB value of the system, so that a stable NE is formed. Hence HLB value $>10$ has been chosen in the formation of o/w type NE. The HLB of tween 80 is $16.7 \&$ that of ethanol is 4.2. Hence the HLB of Smix obtained was $>10$. Further, the co-surfactant ethanol has got good miscibility with the oil phase. Next, the concentration of surfactant was optimized to $10 \% \mathrm{w} / \mathrm{v}$ since, too low $\&$ too much surfactant concentration influences NE stability. The ratio of Smix was found to be important in obtaining transparent NE. Here a ratio of 3:2 was fixed from the experiment. Further the oil \& Smix ratio was optimized for different ratios ranging from 1:9 to $9: 1$. The $1: 5$ ratio of oil \& Smix has led to the formation of stable transparent NE, whereas the 1:6 \& 1:3 ratios of Smix had resulted in less stable NE formulation.

The smallest droplet size $84.45 \mathrm{~nm}$ with a PDI value of 0.197 was obtained for the formulation NE5. This small size might have arrived due to the high energy emulsification preparation technique parameters particularly the sonication time. The time of sonication is important as the effectiveness of emulsifying agents increases with increasing the mixing time, however prolongation of time above certain period may decrease their effectiveness by dropping out from the liquid interface ${ }^{12}$. A small size of NE is very much prerequisite for molecule delivery across skin as the oil droplets tend to provide a channel for antigen delivery ${ }^{13}$. A slight increase in size was obtained for the NE incorporated with antigen i.e (PTAg-NE5) which was determined as $99.42 \mathrm{~nm}$ (Figure $4 \mathrm{f}$ ). The size \& its distribution chart obtained for the NE are given in Figure 4. The size of the nanoemulsions had ranged from $84.45 \mathrm{~nm}$ to $231.4 \mathrm{~nm}$ with narrow distribution as dictated from the value of polydispersity index (PDI) that was $<0.2$.

Emulsifiers, here Smix not only act as an interfacial barrier between oil \& water but also through formation of surface charges called zeta potential, which can produce repulsive electrical forces among approaching oil droplets hinders coalescence ${ }^{14}$. Hence, the value of zeta potential is an indicator of NE physical stability. However, values greater than $-10 \mathrm{mV}$ shows high degree of their stability. It could be said that the formulation NE5 was stable as found from its zeta potential value of $-5.23 \mathrm{mV}$ (Figure 5) that controls the droplets interaction within a NE. The charges might have been arised due to the adsorption of traces of ions from the aqueous phase \& ethanol. The effect of tweens on charge may be less as because these are non-ionic type of emulsifying agents.

The estimated $\mathrm{pH}$ values were ranged from 5.92 to 6.83 , a slightly acidic nature made the $\mathrm{NE}$ favorable for topical administration as it's a non skin irritating $\mathrm{pH}$. Moreover this $\mathrm{pH}$ range matches with the reports of Hepatitis B antigen delivery through nasal mucosa using stabilized nanoemulsion ${ }^{2}$. The viscosity values elicited by all the NE preparations were found to be less than one. This indicates the dilute nanoemulsion which could allow for thorough mixing of antigen with the NE before administration. Again the viscosity of oil, composition of $\mathrm{NE}$ and preparation technique may perhaps influences the NE viscosity. Further, the dilute nature of NE would help in its 
conversion into topical gel state for application over skin. The reduced value of surface tension was found to be constant for all the NE formulations. This might be due to the action of added Smix in the developed nanoemulsion. The results are in accordance with the earlier reports of NE for antigen delivery and confirms the suitability of the presently developed formulation for the topical /transdermal delivery of incorporated antigen. A low value of electrical conductivity shown might be the reason for ions present in the dispersion medium and also the integrity of other components such as soybean oil after NE preparation. Generally conductivity measurements are made to identify the occurrence of any phase inversion in emulsion after subjecting to various process conditions.

TEM image of PTAg-NE5 (Figure 6) showed the incorporation of pertusis antigen in NE5. The nanoemulsion PTAg-NE5 appears dark and the surroundings are bright. A positive image was observed under TEM. Some droplet sizes were measured during TEM analysis, as it is capable of point-to-point resolution. The measured droplet size was in agreement with the results obtained from droplet size analysis using Zetasizer. The photograph specifies the nano globules and its morphology clearly.

The formulation NE5 was found to be physically stable up to a study period 7 days. The accelerated stability results are tabulated in table.3. There was no phase separation took place after centrifugal stability testing. Centrifugation was adopted to find out the short term stability of NE before going for a long term stability analysis. This also helps in the selection of more stable preparation for further development and characterization.

In case of long term stability analysis, the samples were monitored for any significant change with respect to their $\mathrm{pH}$ value as because $\mathrm{pH}$ changes point out the incidence of any

Table 1: Formulation composition of nano emulsion (NE)

\begin{tabular}{|c|c|}
\hline Ingredients & $\mathbf{\%}$ \\
\hline Soybean oil & 2 \\
\hline $\mathrm{S}_{\text {mix }}$ & 10 \\
\hline Water & 88 \\
\hline
\end{tabular}

chemical reactions that may disturb the quality of the preparation. Mostly emulsions formed with vegetable oils may encompass a decrease in $\mathrm{pH}$ due to the hydrolysis of fatty acid esters into free fatty acid degradation products ${ }^{15}$. Here the $\mathrm{pH}$ values of NE5 remained around 6.0 and there was no much difference noted at all temperature conditions. This is an acceptable, non-skin irritating $\mathrm{pH}$ value suitable for vaccine incorporation. The small proportion of oil and its protection as internal phase in the NE might have sheltered its degradation during storage.

The electrical conductivity of NE5 remained unchanged at all storage conditions indicates that there was no phase inversion taken place. Conductivity rest on the concentration of ions in the media and $\mathrm{o} / \mathrm{w}$ emulsion displays conductivity than w/o emulsion.

The droplet size and zeta potential values showed no significant difference at the end of 7 days reflects the physically stable NE. The PDI value of the stored samples were found to be $<0.2$. This reveals that the emulsion with a fairly uniform size distribution is more stable. However a slight increase in size of the internal oil globules might have resulted from the disturbances in electrical double layer by aqueous ions \& the double layer formation by non-ionic emulsifying agents may not be strong enough when compared to the double layers made up of ionic emulsifying agents ${ }^{12}$.

However the formulation stored at $5 \pm 2 \quad$ was much more stable with respect to size analysis compared with other storage conditions. The zeta potential value did not get altered significantly $(\mathrm{P}<0.05)$ when stored at different storage conditions indicates the formation of stable NE.

Table.2: Formulation composition of nano emulsion containing antigen (PTAg-NE5)

\begin{tabular}{|c|c|}
\hline Ingredients & Quantity for 20ml \\
\hline Antigen $(\mathrm{ml})$ & 2 \\
\hline Soybean Oil $(\mathrm{ml})$ & 0.4 \\
\hline Tween $80(\mathrm{ml})$ & 1.6 \\
\hline Ethanol $(\mathrm{ml})$ & 0.4 \\
\hline Water $(\mathrm{ml})$ & 14.6 \\
\hline Phosphate Buffer Saline $(\mathrm{ml})$ & Quantity sufficient \\
\hline
\end{tabular}

Table 3: Properties of nanoemulsion formulations NE1, NE2, NE3, NE4 AND NE5

\begin{tabular}{|c|c|c|c|c|c|}
\hline Property & NE1 & NE2 & NE3 & NE4 & NE5 \\
\hline Droplet size (nm) & $231.4 \pm 3.2$ & $211.8 \pm 2.8$ & $117.1 \pm 2.5$ & $94.12 \pm 3.0$ & $84.45 \pm 2.1$ \\
\hline $\mathrm{pH}$ & $6.51 \pm 0.02$ & $6.21 \pm 0.01$ & $6.83 \pm 0.09$ & $5.92 \pm 0.16$ & $6.50 \pm 0.05$ \\
\hline Viscosity (Poise) & $0.829 \pm 0.042$ & $0.865 \pm 0.031$ & $0.873 \pm 0.06$ & $0.861 \pm 0.04$ & $0.852 \pm 0.056$ \\
\hline Conductivity (ohm) & $0.09 \pm 0.005$ & $0.10 \pm 0.006$ & $0.20 \pm 0.004$ & $0.11 \pm 0.004$ & $0.15 \pm 0.002$ \\
\hline Surface Tension (dyne/cm) & $23.43 \pm 1.5$ & $23.97 \pm 2.0$ & $25.01 \pm 1.7$ & $24.08 \pm 0.09$ & $23.01 \pm 1.02$ \\
\hline
\end{tabular}

Values are mean \pm S.D, $\mathrm{n}=6$.

Table 4: Accelerated stability determination of NE5 after storage at various temperature and at various time periods

\begin{tabular}{|c|c|c|c|c|c|}
\hline Sl.no & \multicolumn{2}{|c|}{ Storage condition/ property } & $\mathbf{2 5} \pm \mathbf{2}$ & $\mathbf{4 0} \pm \mathbf{2}$ & $\mathbf{5} \pm \mathbf{2}$ \\
\hline \multirow{2}{*}{1} & \multirow{2}{*}{ Day 1 } & $\mathrm{pH}$ & $6.51 \pm 0.04$ & $6.72 \pm 0.08$ & $6.45 \pm 0.01$ \\
\cline { 3 - 6 } & & Conductivity (ohm) & $0.11 \pm 0.008$ & $0.20 \pm 0.006$ & $0.15 \pm 0.004$ \\
\hline \multirow{2}{*}{2} & \multirow{2}{*}{ Day 3 } & $\mathrm{pH}$ & $6.50 \pm 0.05$ & $6.70 \pm 0.06$ & $6.42 \pm 0.02$ \\
\cline { 3 - 6 } & & Conductivity (ohm) & $0.10 \pm 0.005$ & $0.19 \pm 0.004$ & $0.16 \pm 0.006$ \\
\hline \multirow{2}{*}{3} & \multirow{2}{*}{ Day 7 } & $\mathrm{pH}$ & $6.21 \pm 0.06$ & $6.65 \pm 0.05$ & $6.40 \pm 0.02$ \\
\cline { 3 - 6 } & & Conductivity (ohm) & $0.11 \pm 0.003$ & $0.23 \pm 0.007$ & $0.10 \pm 0.004$ \\
\cline { 3 - 6 } & & Droplet size (nm) & $121.3 \pm 2.30$ & $143.3 \pm 1.94$ & $112.7 \pm 2.06$ \\
\cline { 3 - 6 } & & Zeta potential (mV) & $-8.46 \pm 0.02$ & $-9.59 \pm 0.04$ & $-8.01 \pm 0.03$ \\
\hline
\end{tabular}

Values are mean \pm S.D, $n=6$ 


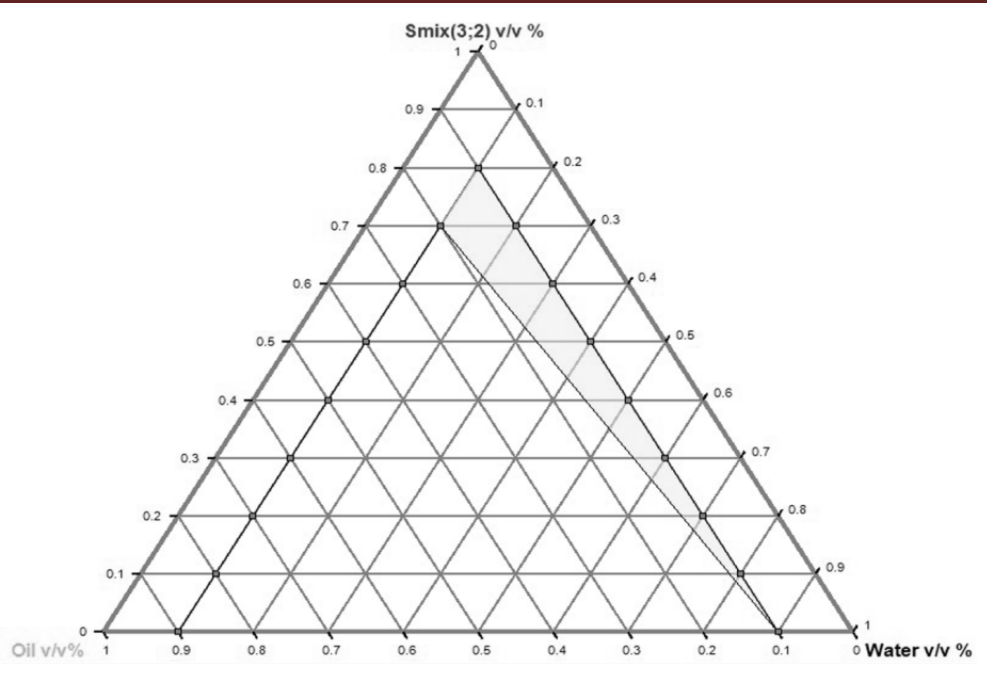

Figure 1: Ternary diagram showing the region of nanoemulsion formation

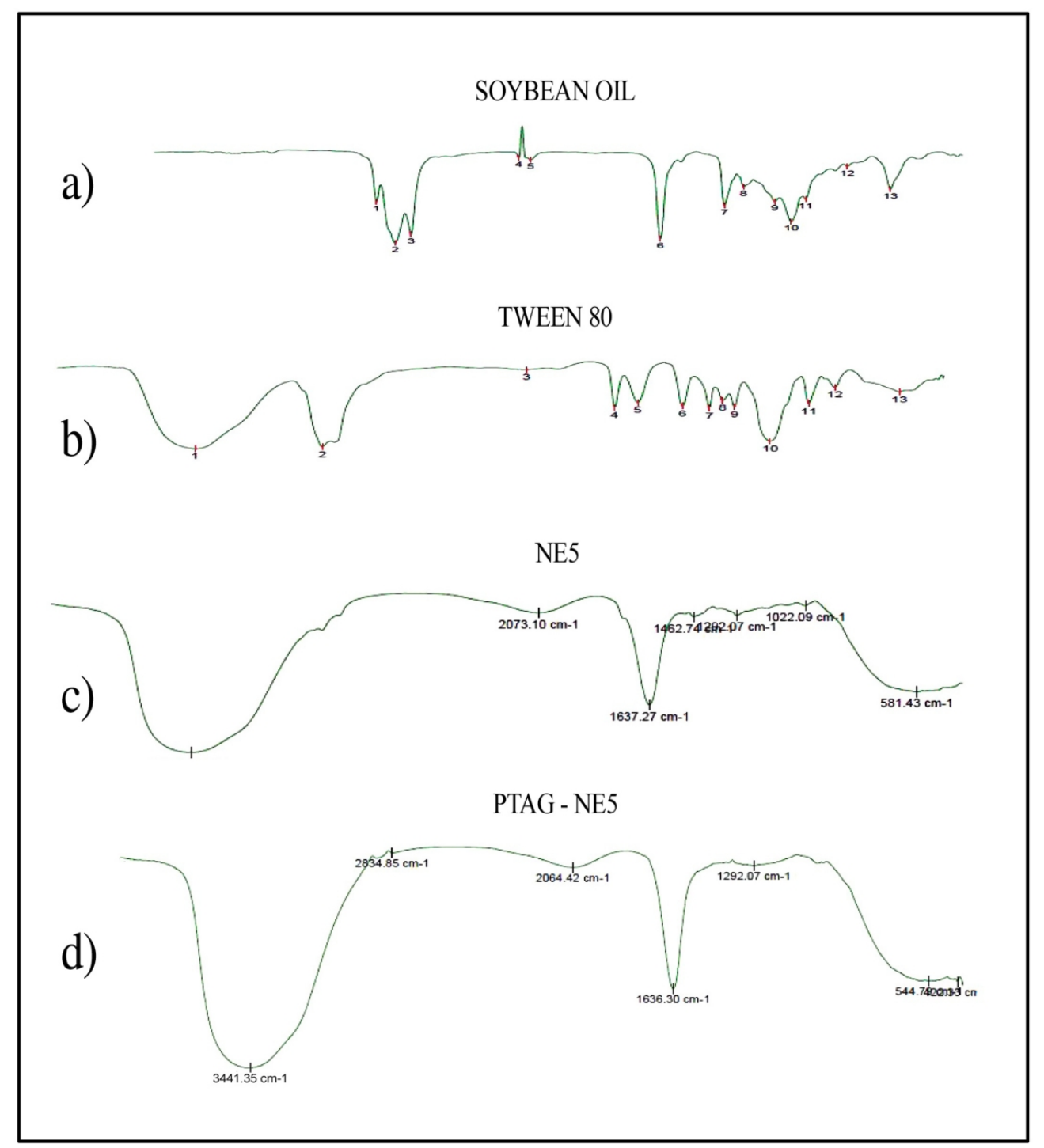

Figure 2: Fourier Transform Infrared spectra of (a) Soybean oil, (b) Tween 80, (c) NE5, (d) PTAg-NE5 


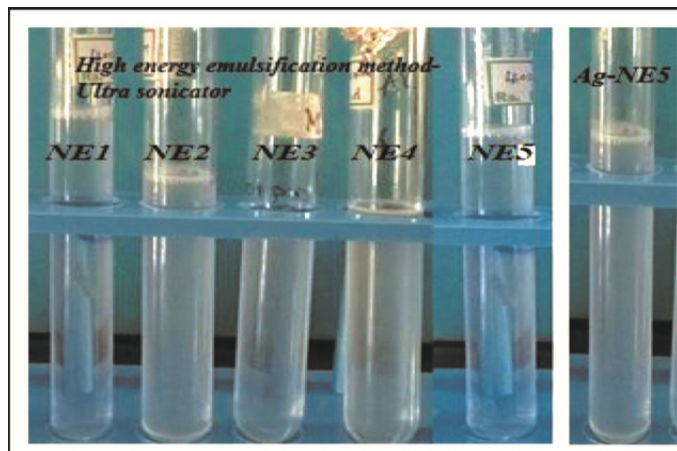

Fig-3: Photograph of Nanoemulsion Formulations

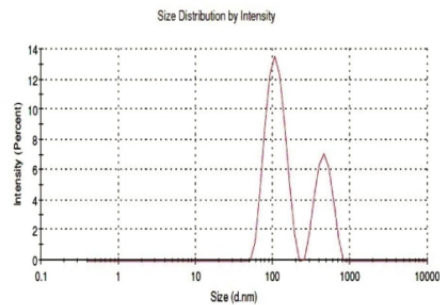

Fig(a): NE - 1

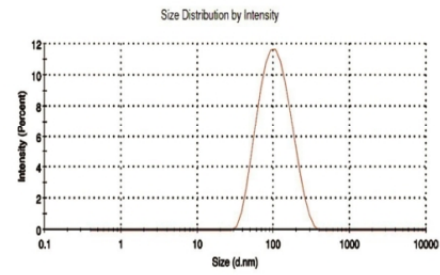

Fig(c): NE -3

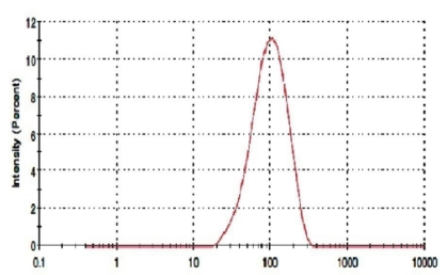

Fig(e): NE - 5

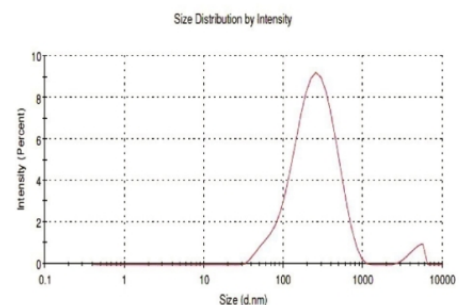

Fig(b): NE - 2

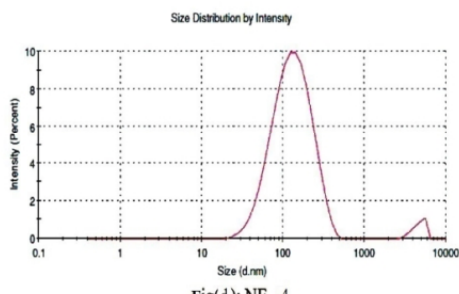

Fig(d): NE - 4

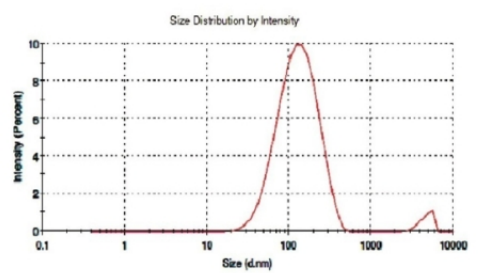

Fig(f): PTAG - NE5

Figure 4: Size of nano emulsion formulations (a) NE1, (b) NE2, (c) NE3, (d) NE4, (e) NE5, (f) PTAg-NE5

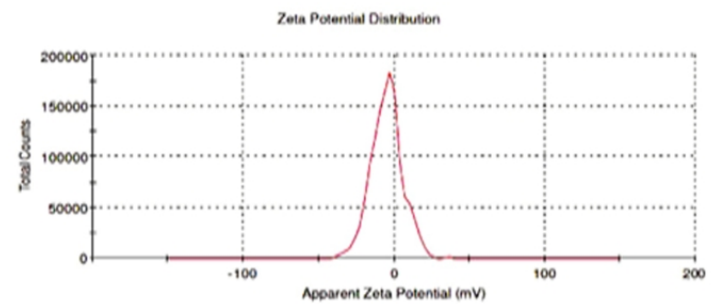

Figure 5: Zeta potential nano emulsion NE5

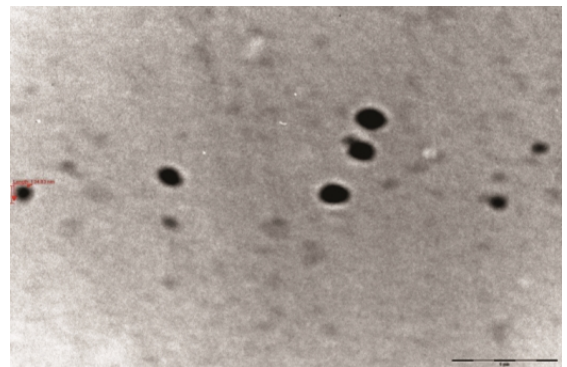

Figure 6: TEM image of pertusis nanoemulsion PTAgNE5 


\section{CONCLUSION}

An o/w type nanoemulsion containing nano sized droplets was prepared successfully by high energy emulsification technique and are found to be physically stable at the conditions tested. The NE was loaded effectively with the pertussis toxin and thus suitable for efficient topical immunization.

\section{ACKNOWLEDGEMENT}

The authors would like to thank National Facility on Drug Development for academia, Pharmaceutical and allied industries for utilizing facilities to carry out the work. The authors also wish to acknowledge Pasteur Institute of India, Coonoor for providing pertussis toxin for the study.

\section{REFERENCES}

1. Mi-Gyeong Kim, Joo Yeon Park, Yuna Shon, Gunwoo Kim, Gayong Shim, Yu-Kyoung Oh. Nanotechnology and vaccine development. Asian Journal of Pharmacutical Science 2014; 9(5):227-235

2. Makidon PE, Nigavekar SS, Bielinska AU, Mank N, Shetty AM, Suman J, et al. Characterization of Stability and Nasal Delivery Systems for Immunization with NanoemulsionBased Vaccines. Journal of Aerosol Medicine and Pulmonary Drug Delivery 2010; 23(2): 77-89.

3. Tri Suciati, Aldy Aliyandi, Satria Di. The development of transdermal nanoemulsion formulation for simultaneous delivery of protein vaccine and artin-M adjuvant. International Journal of Pharmaceutical Science 2014; 6(6): 536-546.

4. Ledet G, Pamujula S, Walker V, Simon S, Graves R, Mandal TK. Development and invitro evaluation of a nanoemulsion for transcutaneous delivery. Drug Development and Industrial Pharmacy 2014; 40(3): $370-$ 379.

5. Hamouda T, SutcliffeJA, Ciotti S, Baker JR Jr. Intranasal immunization of ferrets with commercial trivalent influenza vaccines formulated in a nanoemulsion-based adjuvant, Clinical Vaccine Immunology 2011;18(7): 1167-1175. Epub 2011 May 4.

6. Bouchemal K, Briancon S, Perrier E, Fessin H. Nanoemulsion formulation using spontaneous emulsification: solvent, oil and surfactant optimization. International Journal of Pharmacy 2004; 280(1-2): 241-251.

7. Thomas Delmas, Helene Piraux, Anne-Claude Couffin, Isabelle Texier, Franc-oise Vinet et al. How to prepare and stabilize very small nanoemulsions, Langmuir 2011; 27(5):1683-1692.

8. Chime SA, Kenechukwu FC and Attama AA. Nanoemulsions-advances in formulation, characterization and applications in drug delivery. In: Nanotechnology and Nanomaterials "Application of Nanotechnology in Drug Delivery", edited by Ali Demir Sezer, ISBN 978-953-511628-8, Published: July 25, 2014.

9. Eid Ahmed Mustafa Masoud, Baie Saringat Haji, Arafat Osama Mohammed. The influence of Sucrose ester Surfactants and different storage conditions of the preparation of a Novel Swietenia Macrophylla oil Nano emulsion. International Reseach Journal of Pharmacy 2012; 3(6):2230 - 8407.

10. Daniela S Bernardi, Tatiana A Pereira, Naira R, Maciel, Josiane Borto loto, Gisely S Viera, Gustavo C Oliveira et al. Formation and stability of oil-in-water nanoemulsions containing rice bran oil: in vitro and in vivo assessments, Journal of Nanobiotechnology 9:44, 2011 Sep 28.

11. Samira sadat abolmaali, Ali Mohammad Tamadddon, Fakhr Sadat Farvadi, Saeed Daneshamuz, and Hamidrwza Mogimi.. Pharmaceutical Nanoemulsions and their potential topical and transdermal applications. Iranian Journal of Pharmaceutical Science 2011;7(3):139-150.

12. Barkat Ali Khan, Naveed Akhtar, Haji Muhammad Shoaib Khan, Khalid Waseem, Tariq Mahmood, Akhtar Rasul et al. Basics of pharmaceutical emulsions: A review, African Journal of Pharmacy and Pharmacology 2011; 5(25): 27152725 .

13. Jignesh D Modi, Jayvadan K Patel. Nanoemulsion-Based Gel Formulation of Aceclofenac for Topical Delivery. International Journal of Pharmacy and Pharmaceutical Science Research 2011; 11(1): 6-12.

14. Maali A, and Hameed Mosavian MT. Preparation and Application of Nanoemulsions in the Last Decade (20002010). Journal of Dispersion Science and Technology 2013; 34(1): 92-105.

15. Martini E: Cationic Nanoemulsion preparations as delivery systems, Oligonucleotides: Formulation and physicochemical characterization. Dissertation, (Master). University of Rio Grande do Sul, Porto Alegre; 2005

\section{Cite this article as:}

R. Vijaya, G. Aswini and S. Madheshwaran. Development and In vitro characterization of nanoemulsion as adjuvant for the administration of vaccine using a model antigen. Int. Res. J. Pharm. 2016;7(7):43 -49 http://dx.doi.org/10.7897/22308407.07783

Disclaimer: IRJP is solely owned by Moksha Publishing House - A non-profit publishing house, dedicated to publish quality research, while every effort has been taken to verify the accuracy of the content published in our Journal. IRJP cannot accept any responsibility or liability for the site content and articles published. The views expressed in articles by our contributing authors are not necessarily those of IRJP editor or editorial board members. 